\title{
The change of lung function in children with bronchiolitis obliterans syndrome after hematopoietic stem cell transplant
}

\author{
Sungsu Jung ${ }^{1}$, Hee Mang Yoon ${ }^{2}$, Jisun Yoon ${ }^{3}$, Minjee Park ${ }^{2}$, Eun Sang Rhee ${ }^{2}$, Hyery Kim², \\ Kyung Nam Koh ${ }^{2}$, Jin Seong Lee ${ }^{2}$, Ho Joon $\mathrm{Im}^{2}$, and Jinho $\mathrm{Yu}^{2}$ \\ ${ }^{1}$ Pusan National University Yangsan Hospital \\ ${ }^{2}$ Asan Medical Center \\ ${ }^{3}$ Department of Pediatrics, Mediplex Sejong Hospital
}

September 11, 2020

\begin{abstract}
Background: Bronchiolitis obliterans syndrome (BOS) is a life-threatening respiratory complication of allogeneic hematopoietic stem cell transplant (HSCT). Even though a lung function test is crucial in monitoring BOS, little information exists on the test's association with prognosis in children with BOS. Objectives: The purpose of this study was to determine the correlation between changes in lung function after BOS diagnosis and long term outcomes. Methods: A total of 428 children received allogeneic HSCT from January 2006 to December 2017 at Asan Medical Center. Twenty-three of those children (5.4\%) were diagnosed with BOS after allogeneic HSCT, and their clinical data were reviewed. Twenty-one subjects underwent regular pulmonary function tests for 24 months after the occurrence of BOS. Results: Among the 21 children with BOS, eight died, five underwent a lung transplant (TPL), and 15 required oxygen (O2) therapy. The FEV1\% predicted (pred), FVC\% pred, and FEF25-75\% pred were $37.8 \pm 12.7 \%$ (mean \pm SD), $62.2 \pm 16.2 \%$, and $16.4 \pm 9.6 \%$, respectively, upon BOS diagnosis. Changes in the FEV1\% pred were greater in the death and lung TPL group $(-24.8 \pm 22.3 \%)$ than the survival without lung TPL group $(5.7 \pm 21.8 \%)$, and greater in the O2 therapy (-19.4 $\pm 23.4 \%)$ group than the group without O2 therapy (14.2 $\pm 20.0 \%)$ during the first three months after BOS diagnosis. Conclusion: The change of FEV1 during the first three months after BOS correlated with the prognosis including survival, lung transplantation, and $\mathrm{O} 2$ therapy. These results suggest more active intervention in the first three months after BOS diagnosis may be needed to improve prognosis.
\end{abstract}

\section{Introduction}

Bronchiolitis obliterans syndrome (BOS) is a life-threatening complication of allogeneic hematopoietic cell transplant $(\mathrm{HSCT})^{1}$. It is characterized by an irreversible small airway obstruction caused by epithelial injury, subepithelial inflammation, and fibrosis of small airways ${ }^{2}$. The prevalence of BOS was reported $2 \%$ $6.5 \%$ in allogeneic HSCT recipients in adult cohort ${ }^{3-5}$, and 2.0-2.7\% in pediatric cohorts ${ }^{6,7}$. The prognosis of children with BOS after HSCT is poor; the five-year survival rate is $45-59 \%^{8,9}$. Since the number of patients receiving allogeneic HSCT is increasing, BOS in children will likely increase in the future.

A BOS diagnosis is based on declining $\mathrm{FEV}_{1}$, which is measured serially after $\mathrm{HSCT}^{10}$, and confirmed with pathology of constrictive bronchiolitis or high-resolution computed tomography (CT) showing air trapping, small airway thickening, or bronchiectasis ${ }^{11}$. After BOS diagnosis, pulmonary functions are measured regularly to monitor treatment efficacy and prognosis ${ }^{12}$. Systemic steroids have been the backbone of BOS therapy ${ }^{13-17}$ and inhaled fluticasone, azithromycin, and montelukast (FAM) have been recently introduced ${ }^{18}$. However, the prognosis of BOS is still poor.

A study enlisting subjects with BOS in a multicenter prospective trial and a retrospective cohort concluded that $\mathrm{FEV}_{1}$ declined rapidly in the 6 months prior to BOS diagnosis ${ }^{19}$. The current study is one of few 
studies that describe the longitudinal change of lung function after BOS diagnosis in children and clarify the clinical implications of lung function changes on BOS prognosis.

\section{Study design and Methods}

\section{Study participants}

Between January 2006 and December 2017, 428 children received allogeneic HSCT at Asan Medical Center. A detailed description for study participants is provided in the online supplement . In the present study, 21 children met lung function criteria modified from the National Institutes of Health consensus guidelines for

diagnosis of BOS (E-figure 1) ${ }^{11,20,21}$. No subject has concurrent pulmonary infection. In addition, all subjects underwent chest CT scans, and 13 children showed air trapping, small airway thickening, or bronchiectasis. A lung perfusion/ventilation scan was performed on eight children who showed no clear evidence of BOS according to the CT results. Of those eight, five had heterogeneous multiple matched perfusion defects. Lung biopsies were performed on nine children, and constrictive bronchiolitis was confirmed in all nine. Echocardiograms showed that all 21 patients displayed normal heart function at the time of BOS diagnosis.

The median observation period for all subjects after BOS diagnosis was 34.4 months (IQR, 11.5-62.0 months). In our current analysis, pulmonary function tests (PFTs) were gathered during 24 months after BOS diagnosis, and CT values were gathered during 12 months after diagnosis.

All subjects started treatment immediately upon diagnosis. All subjects received 6 to 17 cycles of methylprednisolone (mPD) (i.v. $20 \mathrm{mg} / \mathrm{kg} /$ day for 3 days, with an interval of 1 month) except one subject who died within 6 months after BOS diagnosis. The median mPD pulse therapy period for all subjects was 12.0 months (IQR: 7.5-12.0 months) (E-table 1). A detailed description is provided in the online supplement . Diagnosis and treatment of BOS and lung function monitoring followed the Pediatric Hemato-oncology Division of Asan Medical Center protocol regarding BOS after HSCT, which was developed with the Pediatric Pulmonology Division in 2011.

We divided subjects into two groups according to BOS prognosis and compared the clinical characteristics, lung function parameters, and chest CT findings between the good and poor prognosis groups. The poor prognosis group included patients who had died or required lung TPL (hereafter labeled Group 1) and Group that had required $\mathrm{O}_{2}$ therapy after BOS diagnosis (hereafter labeled Group 3), and the good prognosis group included survivors that did not require lung TPL (hereafter labeled Group 2) or Group that did not require $\mathrm{O}_{2}$ therapy after BOS diagnosis (hereafter labeled Group 4). $\mathrm{O}_{2}$ therapy was defined as $\mathrm{O}_{2}$ therapy for more than 1 day due to dyspnea after BOS diagnosis and $\mathrm{O}_{2}$ therapy was applied when dyspnea was accompanied by less than $90 \%$ of $\mathrm{S}_{\mathrm{P}} \mathrm{O}_{2}{ }^{22}$.

\section{Lung Function Tests}

Detailed descriptions for measuring PFT, IOS, FeNO, and 6MWT are provided in the online supplement

\section{Chest CT}

Patients underwent CT examinations upon BOS diagnosis and 3, 6, and 12 months after diagnosis of BOS using various CT systems (seeonline supplement ).

\section{Statistical analysis}

Statistical analyses were conducted using SPSS version 23 (SPSS, Chicago, IL) (see online supplement ).

\section{Results}

\section{Clinical characteristics}

The clinical characteristics of the subjects are shown in Table 1 . Of the 21 subjects, eight were male and 13 were female. Their age at the time of diagnosis with hemato-oncological diseases was $10.4 \pm 4.7$ years (mean \pm standard deviation), and their age at the time of HSCT was 11.6 \pm 4.1 years. Their age at the time of BOS 
diagnosis was $12.7 \pm 4.0$ years, and the average time to BOS diagnosis after HSCT was $14.0 \pm 10.1$ months (Table 1). Clinical characteristics did not show statistical differences between Groups 1 and 2 and Groups 3 and 4 (Table 2).

\section{Prognosis}

Eight subjects $(8 / 21,38.1 \%)$ died; six $(6 / 21,28.6 \%)$ died due to respiratory failure after BOS diagnosis, one died from fungal infection, and one died from acute renal failure. The mean time before death after BOS diagnosis was $18.6 \pm 15.1$ months. Five subjects $(5 / 21,23.8 \%)$ underwent lung transplantation, and the mean time before lung transplantation after BOS diagnosis was $30.4 \pm 28.0$ months. In addition, 15 subjects $(15 / 21$, $71.4 \%$ ) underwent $\mathrm{O}_{2}$ therapy, and the mean time before $\mathrm{O}_{2}$ therapy after BOS diagnosis was $17.6 \pm 19.8$ months (Table 1). We used $\mathrm{O}_{2}$ therapy and death or lung TPL to analyze the prognosis of BOS.

\section{Lung function during the study period}

Data gathered after lung TPL were excluded because they did not represent the original characteristics of the subjects. E-figure 1 shows the change in lung function caused by BOS. $\mathrm{FEV}_{1} \%$ pred, FVC\% pred and $\mathrm{FEF}_{25-75} \%$ pred showed statistical significance during study period in linear mixed modeling, and $\mathrm{FEV}_{1} \%$ pred, $\mathrm{FVC} \%$ pred, and $\mathrm{FEF}_{25-75} \%$ pred decreased significantly upon BOS diagnosis compared to pre-HSCT $(P<0.001)$. FEV\% pred, $\mathrm{FVC} \%$ pred, and $\mathrm{FEF}_{25-75} \%$ pred were $92.8 \pm 13.0 \%, 87.9 \pm 14.7 \%$, and $113.0 \pm 24.2 \%$, respectively, before $\mathrm{HSCT}$, and $\mathrm{FEV}_{1} \%$ pred, $\mathrm{FVC} \%$ pred, and $\mathrm{FEF}_{25-75} \%$ pred at the time of BOS diagnosis were $37.8 \pm 12.7 \%, 62.2 \pm 16.2 \%$, and $16.4 \pm 9.6 \%$, respectively. Among these values, $\mathrm{FEF}_{25-75} \%$ pred decreased the most from pre-HSCT $(111.7 \pm 24.2 \%)$ to BOS diagnosis $(16.0 \pm 3.3 \%)$. After BOS diagnosis, $\mathrm{FEV}_{1} \%$ pred, $\mathrm{FVC} \%$ pred, and $\mathrm{FEF}_{25-75} \%$ pred were significantly reduced at each time point with an interval of 3 months during study period when compared to pre-HSCT (E-table 2).

\section{Comparison of lung function during the study period according to the prognosis}

Twenty-one subjects repeated PFTs regularly for 24 months after BOS occurred. Among them, nine children did not complete PFTs because they are died or underwent lung TPL within 24 months after BOS diagnosis. The events were defined as death or lung TPL, and $\mathrm{O}_{2}$ therapy. The median pre-event period for PFTs in all subjects was 24.0 months (interquartile range [IQR] 7.5-24.0 months) (E-table 1).

Figure 1 illustrates the comparison of lung function during the study period between Group 1 versus Group 2 and Group 3 versus Group 4. $\mathrm{FEV}_{1} \%$ pred, $\mathrm{FVC} \%$, pred, and $\mathrm{FEF}_{25-75} \%$ pred were lower in Group 1 than in Group 2 at all time points after BOS diagnosis. However, these differences were significant at only the 6, 9, and 18 month time points for $\mathrm{FEV}_{1} \%$ pred and the 9 month time point for $\mathrm{FEF}_{25-75} \%$ pred after BOS diagnosis (all $P<0.05$, Figure 1a, E-table 3a). When comparing Groups 3 and $4, \mathrm{FEV}_{1} \%$ pred and FVC\% pred were significantly lower in Group 3 than in Group 4 at 3 months post-BOS diagnosis and afterward. The levels of $\mathrm{FEF}_{25-75} \%$ pred were also significantly lower in Group 3 than in Group 4 at 3, 6, 9, 15, 18, and 21 months after BOS diagnosis (all $P<0.05$, Figure 1b, E-table 3b).

\section{Lung function change during the study period according to the prognosis}

We compared changes in $\mathrm{FEV}_{1} \%$ pred between Groups 1 and 2 and between Groups 3 and 4 during the study period (Table 3). A detailed description for the calculation is provided in theonline supplement . There was no significant difference in $\mathrm{FEV}_{1} \%$ pred change from pre-transplant to BOS diagnosis between Groups 1 and 2 and Groups 3 and 4 . We compared changes in $\mathrm{FEV}_{1} \%$ pred at each time point with an interval of 3 months after BOS diagnosis during the study period, and there was a significant difference between Group $1(-24.8 \pm 22.3 \%)$ and Group $2(5.7 \pm 21.8 \%)(P=0.016)$ and between Group $3(-19.4 \pm 24.4 \%)$ and Group 4 $(8.6 \pm 21.9 \%)(P=0.041)$ during the first 3 months after BOS diagnosis. Likewise, there was a significant difference in the change of $\mathrm{FEF}_{25-75 \%}$ pred between Group $1(-32.4 \pm 23.6 \%)$ and Group $2(11.7 \pm 37.1 \%)$ $(\mathrm{P}=0.007)$ and between Group $3(-19.4 \pm 24.4 \%)$ and Group $4(16.8 \pm 39.6 \%)(\mathrm{P}=0.026)$ during the first 3 months after BOS diagnosis (E-table 4a). Detailed descriptions for additional analysis are provided in the online supplement (E-table $4 \mathrm{~b})$. 
The prognosis of BOS according to the slope of $\mathrm{FEV}_{1}$ change during the first 3 months after BOS diagnosis

When death or lung TPL was defined as an event, the median pre-event period 13.5 months (IQR, 8.4-30.9 months) for Group 1 after BOS diagnosis, and the observation period for Group 2 was 59.7 months (IQR, 43.0-85.6 months). When $\mathrm{O}_{2}$ therapy was defined as an event, 10.2 months (IQR, 5.8-17.9 months) for Group 3, and the observation period for Group 4 was 59.8 months (IQR, 33.9-102.0 months).

When comparing the Kaplan-Meier estimates between groups with positive and negative slopes of $\mathrm{FEV}_{1}$ change during the first 3 months after BOS diagnosis, the group with a negative slope had a higher likelihood of being Group 1 with a marginal significance when compared to the group with a positive slope $(P=0.057$ according to the log-rank test) (Figure 2a). Also, the group with a negative slope of $\mathrm{FEV}_{1}$ change during the first 3 months after BOS diagnosis had a significantly higher probability of being Group 3 when compared to the group with a positive slope ( $P=0.014$ according to the log-rank test) (Figure $2 \mathrm{~b}$ ).

The occurrence of air trapping on chest CTs during the first 12 months after BOS diagnosis according to the prognosis

We compared changes in air trapping percent at each time point during the first 12 months after BOS diagnosis. Detailed description for the calculation and analysis are provided in the online supplement .

\section{Airway resistance and reactance, FeNO, and 6MWT according to prognosis}

We compared IOS, FeNO, and 6MWT values upon BOS diagnosis. Detailed description for the analysis are provided in the online supplement .

\section{Discussion}

A major finding regarding BOS is the decrease of lung function, especially reduced airway function including $\mathrm{FEV}_{1}$ and $\mathrm{FEF}_{25-75} \%$. However, no study has examined the relationship between prognosis and lung function change in patients with BOS. Our current study is the first to discover the clinical implications of lung function change in BOS, especially during the first 3 months after BOS diagnosis. In the current study, 21 children were diagnosed with BOS, treated, and monitored with regular PFTs prospectively on the basis of our protocol, and accumulated data were analyzed retrospectively. The good prognosis groups (Groups 2 and 4) showed different changes in $\mathrm{FEV}_{1} \%$ pred and $\mathrm{FEF}_{25-75} \%$ pred during the first 3 months after BOS diagnosis when compared to the poor prognosis groups (Groups 1 and 3).

Because BOS is characterized by the narrowing of small airways via a fibroproliferative process ${ }^{23,24}$, the reduction in $\mathrm{FEV}_{1}$ and $\mathrm{FEF}_{25-75} \%$ may be more prominent than in $\mathrm{FVC}$, as in our current analysis. In a cohort study of adult patients with BOS, lung function trajectory was examined, and it concluded that $\mathrm{FEV}_{1}$ decreased rapidly 6 months before BOS diagnosis and stabilized after BOS diagnosis ${ }^{19}$. Also, a study examining BOS after lung transplantation ${ }^{25}$ concluded that subjects with higher $\mathrm{FEV}_{1}$ or $6 \mathrm{MWT}$ values (measured at 3,6, and 12 months and annually after lung transplantation) had a better chance of survival and a lower risk of developing BOS. However, these studies focused on pulmonary function before BOS diagnosis and did not show a relationship between lung function trajectory after BOS diagnosis and BOS prognosis. In the current study, we analyzed spirometric parameters over time after BOS diagnosis, and we investigated impulse oscillometric values, 6MWT and FeNO values at BOS diagnosis.

The pathogenesis of BOS after HSCT is associated with alloreactivity (e.g. graft-versus-host disease) ${ }^{26,27}$, and systemic steroids and other anti-inflammatory agents are typically used to treat BOS; however, peribronchial inflammation can still progress into fibrosis causing a poor prognosis ${ }^{18}$. Our current study showed that a rapid decline in $\mathrm{FEV}_{1}$ in the first 3 months after BOS correlates with poor prognosis (i.e. death or the need for a lung transplant or $\mathrm{O}_{2}$ therapy). Moreover, the slope of $\mathrm{FEV}_{1} \%$ pred level change during the first 3 months after BOS diagnosis determined BOS prognosis in Kaplan-Meier estimates even though all patients with BOS received the same treatment. Our first hypothesis that may explain this phenomenon is that airway inflammation in patients with a rapid $\mathrm{FEV}_{1}$ decline may be too severe to be controlled by anti-inflammatory 
treatment. Our second hypothesis posits that the rate of lung function reduction after BOS development in some patients is faster because it rapidly progresses into fibrosis in the airway, which can no longer respond to anti-inflammatory treatment. This progression may depend on an individual's susceptibility to fibrosis after injury in the airway ${ }^{28}$. This study is the first to show that changes in pulmonary function during early disease processes can predict BOS prognoses. New treatment modalities during the first 3 months after BOS diagnosis are needed for patients with a poor prognosis.

This study found different changes in $\mathrm{FEV}_{1} \%$ pred and $\mathrm{FEF}_{25-75} \%$ pred during the first 3 months after BOS diagnosis between good and poor prognosis groups. However, changes in PFTs could not be compared across all periods because of death or lung TPL. Lung function likely declines persistently in patients with a poor prognosis, and if their data were included, a significant difference in the change of $\mathrm{FEV}_{1} \%$ pred after the first 3 months after BOS would likely occur. However, the data from subjects with a poor prognosis (Groups 1 and 3) were all present except for one subject until 6 months after BOS diagnosis. Furthermore, PFT changes from 3 to 6 months after BOS diagnosis were not significant. Therefore, the first 3 months after BOS diagnosis should be considered a meaningful period for determining prognosis. In addition, Figure 1a shows that $\mathrm{FEV}_{1}$ in Group 1 tended to increase after 18 months from BOS diagnosis, but not in a significant manner. However, this analysis was distorted because subjects with low lung functions died early. In this group, only two subjects completed PFTs during the 24 months after BOS diagnosis, and others dropped out due to death or lung TPL within 24 months after BOS diagnosis. We analyzed the PFT trajectories of two subjects who consistently performed PFTs until 24 months after BOS diagnosis (E-table 5, E-figure 3 ). Both subjects eventually received lung TPL because of decreasing lung function. One received lung TPL at 34.4 months after BOS diagnosis and the other received lung TPL at 68.5 months after BOS diagnosis. E-figure 3 illustrates that $\mathrm{FEV}_{1}$ steadily decreased during the first 24 months after BOS diagnosis in both subjects.

In this study, subjects performed IOS upon BOS diagnosis, and we found that the Xrs5\% value in Group 3 was significantly higher than in Group 4 . Reactance implies tissue elastance and inertance, especially at lower frequencies (e.g. $5 \mathrm{~Hz}$ ), with a close association with capacitance, which better reflects the elasticity of the lung periphery ${ }^{29}$. No study has clarified the effect of IOS on the prognosis of BOS after HSCT. Studies using patients with diffuse interstitial lung disease and emphysema showed pulmonary fibrosis and emphysema can cause changes in Xrs5\% pred due to lung stiffness, hyperinflation and a loss of lung elastic recoil, respectively ${ }^{30,31}$. Another study examining children with postinfectious bronchiolitis obliterans (PIBO) showed greater differences in Xrs5 than Rrs5 in children with PIBO compared to other groups ${ }^{32}$. These findings are consistent with our current findings, and it suggests that Xrs5\% pred may better reflect small airway obstruction than Rrs5\% pred due to peribronchial fibrosis of lung periphery in children with $\mathrm{PIBO}$ and BOS ${ }^{29-31}$. This study showed that IOS can predict BOS prognosis in young children who cannot undergo spirometry.

We examined CT results, which are another crucial factor in diagnosing BOS. Some studies attempted to predict lung function via CT scan in BOS patients, but this is challenging in young children because no consensus exists regarding reference values that define air trapping in chest CTs. In a pilot feasibility study of children (6-17 years of age), quantitative computed tomography assessments in children with BOS showed a correlation between their lung function and air trapping as defined by the individualized threshold ([attenuation values of normal lung parenchyma + attenuation values of air trapping area] $/ 2)^{33}$. In our current analysis, we used this as a cut-off value to distinguish air trapping lungs from normal lungs on CT scans. The percent of air trapping total lung volume according to CT measurements (air trapping volume/total lung volume) at 3, 6, and 12 months after BOS diagnosis was calculated, and Groups 1 and 2 as well as Groups 3 and 4 were compared. The change in air trapping during the first 3 months after BOS diagnosis was greater in poor prognosis groups (Groups 1 and 3), but the result was not significant. This suggests that the critical period for determining the prognosis of BOS is the first 3 months after BOS diagnosis, which agrees with the analysis of lung function change during BOS. In addition, these results suggest that PFTs are more sensitive to predict the prognosis of BOS even though CT scans may be helpful in tracking the prognosis of BOS. However, we could not perform expiration chest CTs to identify air trapping 
because children with dyspnea had difficulty completing expiration CTs, and frequent radiation exposure was a concern ${ }^{34}$.

The major limitations of the current study are a small sample size and the use of different observation periods for each subject. However, there are very few studies examining pulmonary function in children diagnosed with BOS because of its low prevalence after hematopoietic stem cell transplantation. This is also the first study to show that changes in pulmonary function after BOS diagnosis can predict the prognosis. In the survival analysis, we also showed that PFT changes during the first 3 months after BOS diagnosis in children may influence their prognosis. Furthermore, when the observation periods of all subjects were unified to 24 months after BOS diagnosis in the analysis, there was a significant difference in the change of $\mathrm{FEV}_{1}$ during the first 3 months after BOS diagnosis between Groups 3 and 4 (E-table 6). As another possibility to affect the results, the difference in the diagnosis time of BOS after HSCT or the difference in lung function change before BOS diagnosis among study subjects could be considered to affect the change in lung function after diagnosis of BOS. However, lung function changes during 6 months before BOS diagnosis had no effect on the prognosis (data was not shown).

In conclusion, the change of $\mathrm{FEV}_{1}$ during the first 3 months after BOS diagnosis was significantly different between good and poor prognosis groups. Our current analysis shows that the phase right after BOS diagnosis is the most critical in determining the prognosis of BOS. These results suggest that an active intervention strategy is needed during the first 3 months after BOS diagnosis to improve its prognosis

\section{Figure Legends}

Figure 1. Comparison of lung function during the study period according to the prognosis . (a) Lung function between death or lung transplant (TPL) (Group 1) and survival without lung TPL (Group 2). (b) Lung function between Group with $\mathrm{O}_{2}$ therapy (Group 3) and group without $\mathrm{O}_{2}$ therapy (Group 4). BOS, bronchiolitis obliterans syndrome. ${ }^{*} P<0.05$.

Figure 2. The prognosis of bronchiolitis obliterans syndrome (BOS) according to the slope of FEV $V_{1}$ change during the first 3 months after BOS diagnosis. Group A was defined as subjects with negative slope of FEV1 change during the first 3 months after diagnosis of BOS, and Group B was defined as Subjects with positive slope of FEV1 change during the first 3 months after diagnosis of BOS. (a) Cumulative probability of an event according to the slope of FEV1 change during the first 3 months after BOS diagnosis. Death or lung transplant (TPL) was defined as an event. (b) Cumulative probability of an event according to the slope of FEV1 change during the first 3 months after BOS diagnosis. $\mathrm{O}_{2}$ therapy was defined as event

\section{References}

1. Patriarca F, Skert C, Sperotto A, Damiani D, Cerno M, Geromin A, Zaja F, Stocchi R, Prosdocimo S, Fili C, et al. Incidence, outcome, and risk factors of late-onset noninfectious pulmonary complications after unrelated donor stem cell transplantation. Bone Marrow Transplant 2004;33:751-758.

2. Ralph DD, Springmeyer SC, Sullivan KM, Hackman RC, Storb R, Thomas ED. Rapidly progressive airflow obstruction in marrow transplant recipients. Possible association between obliterative bronchiolitis and chronic graft-versus-host disease. Am Rev Respir Dis 1984;129:641-644.

3. Santo Tomas LH, Loberiza FR, Jr., Klein JP, Layde PM, Lipchik RJ, Rizzo JD, Bredeson CN, Horowitz MM. Risk factors for bronchiolitis obliterans in allogeneic hematopoietic stem-cell transplantation for leukemia. Chest 2005;128:153-161.

4. Marras TK, Chan CK, Lipton JH, Messner HA, Szalai JP, Laupacis A. Long-term pulmonary function abnormalities and survival after allogeneic marrow transplantation. Bone Marrow Transplant 2004;33:509517.

5. Kwok WC, Liang BM, Lui MMS, Tam TCC, Sim JPY, Tse EWC, Leung AYH, Kwong YL, Lie AKW, Ip MSM, et al. Rapid versus gradual lung function decline in bronchiolitis obliterans syndrome after haematopoietic stem cell transplantation is associated with survival outcome. Respirology 2019;24:459-466. 
6. Griese M, Rampf U, Hofmann D, Fuhrer M, Reinhardt D, Bender-Gotze C. Pulmonary complications after bone marrow transplantation in children: twenty-four years of experience in a single pediatric center. Pediatr Pulmonol 2000;30:393-401.

7. Walther S, Rettinger E, Maurer HM, Pommerening H, Jarisch A, Sorensen J, Schubert R, Berres M, Bader $\mathrm{P}$, Zielen $\mathrm{S}$, et al. Long-term pulmonary function testing in pediatric bronchiolitis obliterans syndrome after hematopoietic stem cell transplantation. Pediatr Pulmonol 2020.

8. Ditschkowski M, Elmaagacli AH, Koldehoff M, Gromke T, Trenschel R, Beelen DW. Bronchiolitis obliterans after allogeneic hematopoietic SCT: further insight-new perspectives? Bone Marrow Transplant 2013;48:1224-1229.

9. Nakaseko C, Ozawa S, Sakaida E, Sakai M, Kanda Y, Oshima K, Kurokawa M, Takahashi S, Ooi J, Shimizu T, et al. Incidence, risk factors and outcomes of bronchiolitis obliterans after allogeneic stem cell transplantation. Int J Hematol 2011;93:375-382.

10. Estenne M, Maurer JR, Boehler A, Egan JJ, Frost A, Hertz M, Mallory GB, Snell GI, Yousem S. Bronchiolitis obliterans syndrome 2001: an update of the diagnostic criteria. J Heart Lung Transplant 2002;21:297-310.

11. Jagasia MH, Greinix HT, Arora M, Williams KM, Wolff D, Cowen EW, Palmer J, Weisdorf D, Treister NS, Cheng GS, et al. National Institutes of Health Consensus Development Project on Criteria for Clinical Trials in Chronic Graft-versus-Host Disease: I. The 2014 Diagnosis and Staging Working Group report. Biol Blood Marrow Transplant 2015;21:389-401.e381.

12. Lama VN, Murray S, Lonigro RJ, Toews GB, Chang A, Lau C, Flint A, Chan KM, Martinez FJ. Course of FEV(1) after onset of bronchiolitis obliterans syndrome in lung transplant recipients. Am J Respir Crit Care Med 2007;175:1192-1198.

13. Moran TJ, Hellstrom HR. Bronchiolitis obliterans: an experimental study of the pathogenesis and the use of cortisone in modification of the lesions. AMA Arch Pathol 1958;66:691-707.

14. Moonnumakal SP, Fan LL. Bronchiolitis obliterans in children. Curr Opin Pediatr 2008;20:272-278.

15. Zhang L, Silva FA. [Bronchiolitis obliterans in children]. J Pediatr (Rio J) 2000;76:185-192.

16. Teper A, Fischer GB, Jones MH. [Respiratory sequelae of viral diseases: from diagnosis to treatment]. J Pediatr (Rio J) 2002;78 Suppl 2:S187-194.

17. Tomikawa SO, Adde FV, da Silva Filho LV, Leone C, Rodrigues JC. Follow-up on pediatric patients with bronchiolitis obliterans treated with corticosteroid pulse therapy. Orphanet J Rare Dis 2014;9:128.

18. Williams KM, Cheng GS, Pusic I, Jagasia M, Burns L, Ho VT, Pidala J, Palmer J, Johnston L, Mayer $\mathrm{S}$, et al. Fluticasone, Azithromycin, and Montelukast Treatment for New-Onset Bronchiolitis Obliterans Syndrome after Hematopoietic Cell Transplantation. Biol Blood Marrow Transplant 2016;22:710-716.

19. Cheng GS, Storer B, Chien JW, Jagasia M, Hubbard JJ, Burns L, Ho VT, Pidala J, Palmer J, Johnston L, et al. Lung Function Trajectory in Bronchiolitis Obliterans Syndrome after Allogeneic Hematopoietic Cell Transplant. Ann Am Thorac Soc 2016;13:1932-1939.

20. Williams KM, Chien JW, Gladwin MT, Pavletic SZ. Bronchiolitis obliterans after allogeneic hematopoietic stem cell transplantation. Jama 2009;302:306-314.

21. Filipovich AH, Weisdorf D, Pavletic S, Socie G, Wingard JR, Lee SJ, Martin P, Chien J, Przepiorka D, Couriel D, et al. National Institutes of Health consensus development project on criteria for clinical trials in chronic graft-versus-host disease: I. Diagnosis and staging working group report. Biol Blood Marrow Transplant 2005;11:945-956.

22. Walsh BK, Smallwood CD. Pediatric Oxygen Therapy: A Review and Update. Respir Care 2017;62:645661. 
23. Barker AF, Bergeron A, Rom WN, Hertz MI. Obliterative bronchiolitis. N Engl J Med 2014;370:18201828.

24. Chambers DC. Bronchiolitis obliterans syndrome 'endotypes' in haematopoietic stem cell transplantation. Respirology 2019;24:408-409.

25. Fakhro M, Ingemansson R, Algotsson L, Lindstedt S. Impact of Forced Expiratory Volume in 1 Second (FEV1) and 6-Minute Walking Distance at 3, 6, and 12 Months and Annually on Survival and Occurrence of Bronchiolitis Obliterans Syndrome (BOS) After Lung Transplantation. Ann Transplant 2017;22:532-540.

26. Belperio JA, Weigt SS, Fishbein MC, Lynch JP, 3rd. Chronic lung allograft rejection: mechanisms and therapy. Proc Am Thorac Soc 2009;6:108-121.

27. Weigt SS, Wallace WD, Derhovanessian A, Saggar R, Saggar R, Lynch JP, Belperio JA. Chronic allograft rejection: epidemiology, diagnosis, pathogenesis, and treatment. Semin Respir Crit Care Med 2010;31:189207.

28. Mathai SK, Schwartz DA, Warg LA. Genetic susceptibility and pulmonary fibrosis. Curr Opin Pulm Med 2014;20:429-435.

29. Bickel S, Popler J, Lesnick B, Eid N. Impulse oscillometry: interpretation and practical applications. Chest 2014;146:841-847.

30. van Noord JA, Clement J, Cauberghs M, Mertens I, Van de Woestijne KP, Demedts M. Total respiratory resistance and reactance in patients with diffuse interstitial lung disease. Eur Respir J 1989;2:846-852.

31. Van Noord JA, Clement J, Van de Woestijne KP, Demedts M. Total respiratory resistance and reactance in patients with asthma, chronic bronchitis, and emphysema. Am Rev Respir Dis 1991;143:922-927.

32. Lee E, Yoon J, Cho HJ, Hong SJ, Yu J. Respiratory reactance in children aged three to five years with postinfectious bronchiolitis obliterans is higher than in those with asthma. Acta Paediatr 2017;106:81-86.

33. Kim HG, Shin HJ, Kim YH, Sohn MH, Lyu CJ, Kim MJ, Kim KW, Lee MJ. Quantitative computed tomography assessment of graft-versus-host disease-related bronchiolitis obliterans in children: A pilot feasibility study. Eur Radiol 2015;25:2931-2936.

34. Lucaya J, Garcia-Pena P, Herrera L, Enriquez G, Piqueras J. Expiratory chest CT in children. AJR Am J Roentgenol 2000;174:235-241.

\section{Hosted file}

_2_table only_20200910.docx available at https://authorea.com/users/357969/articles/480306the-change-of-lung-function-in-children-with-bronchiolitis-obliterans-syndrome-afterhematopoietic-stem-cell-transplant
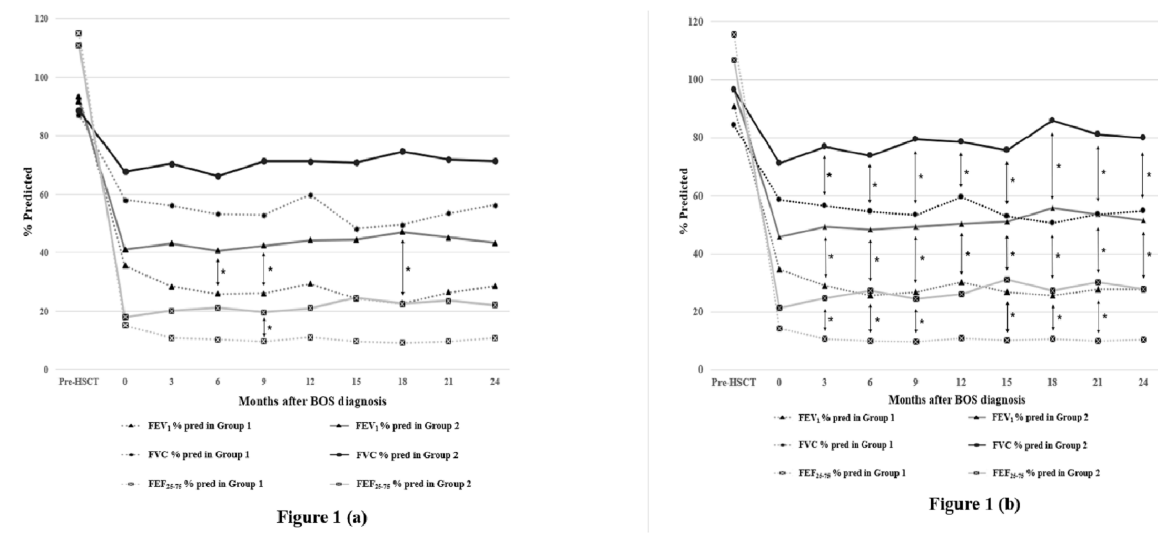


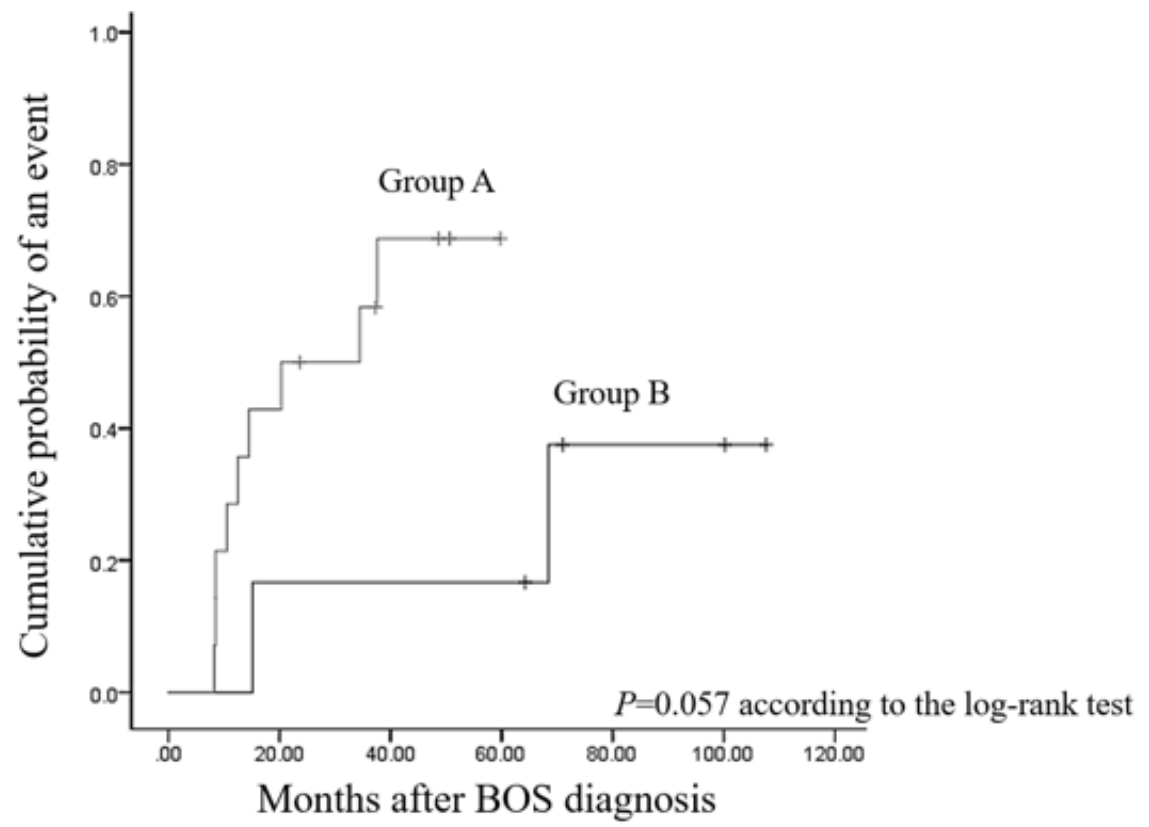

(a)

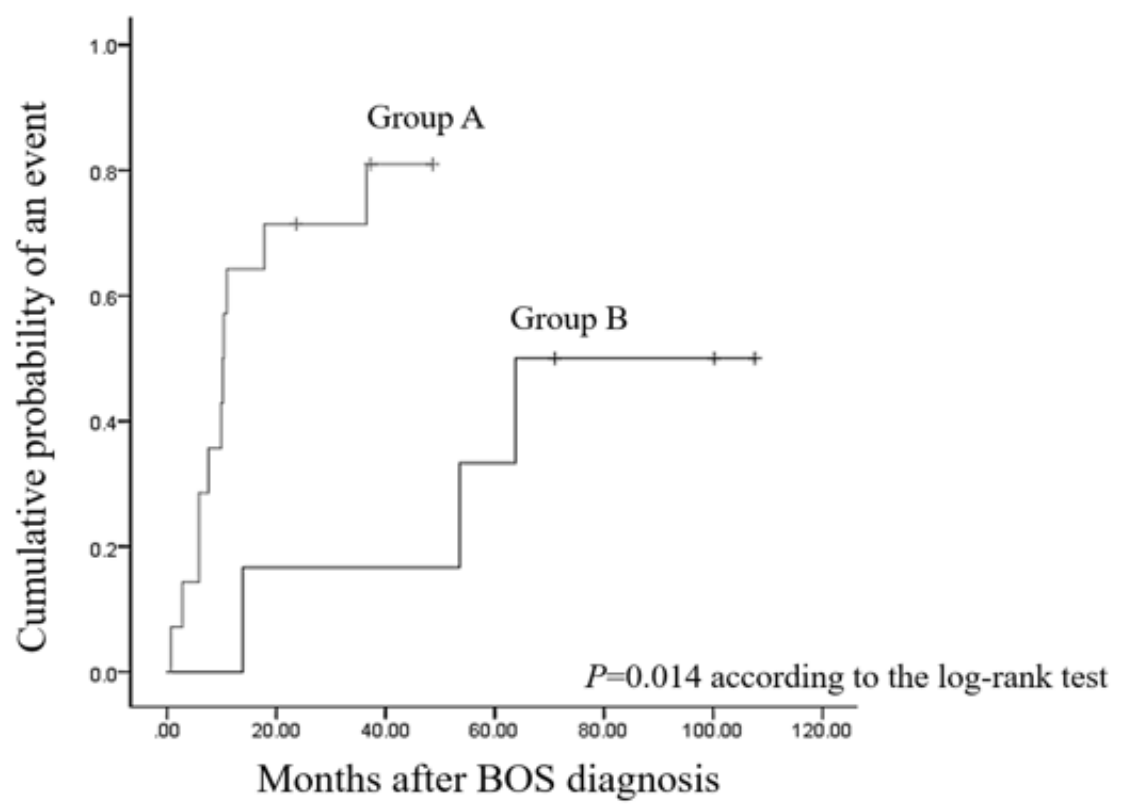

(b)

Figure 2 